\title{
Enzyme Sensors for L-Amino Acid Based on Electrochemical Detection of NADH
}

\author{
Hsien-Chang CHANG*, Akinori UENO, Hiroshi YAMADA, \\ Tomokazu MATSUE and Isamu UCHIDA
}

Received June 11, 1990; Accepted July 28, 1990

\section{INTRODUCTION}

Extensive studies for developing biosensors to detect L-amino acids have been performed in the last decade. The sensors proposed so far were based on gas or ion selective electrodes coupled with amino acid oxidase 1 ). However, since amino acid oxidase has no specificity in the oxidation, it is practically impossible to detect a specific amino acid with these biosensors. In the present paper we describe a new amperometric sensor for determination of individual L-amino acid based on electrochemical regeneration of $\mathrm{NADH}$ catalyzed by diaphorase and L-amino acid dehydrogenase, both of which are coimmobilized on the electrode surface. We applied the two enzymes-immobilized electrodes to the quantitative determination of individual amino acid in a soy sauce. The results showed a good agreement with those obtained by an amino acid analyzer based on high performance liquid chromatography (HPLC).

\section{EXPERIMENTAL}

Diaphorase (Dp, EC 1.6.99.-), L-alanine dehydrogenase (AlaDH, EC 1.4.1.1) and L-leucine dehydrogenase (LeuDH, EC 1.4.1.9) purified from Bacillus stearothermophilus were supplied from UNITIKA Co.. Beef liver L-glutamate dehydro-

Department of Molecular Chemistry and Engineering, Faculty of Engineering, Tohoku University (AramakiAoba, Aoba-ku, Sendai 980, Japan)

Key Words: Diaphorase, L-Amino Acid Dehydrogenase, Immobilized Electrode genase (GluDH, EC 1.4.1.3) was purchased from Oriental Yeast Co.. All the enzymes were used as received. The enzyme coimmobilized electrodes were prepared by mixing the solution containing two enzymes (Dp (62.4 U) + GluDH (40.0 U), Dp $(28.0 \mathrm{U})+\mathrm{AlaDH}(16.0 \mathrm{U})$, and Dp (28.0 U) + LeuDH (11.1 U)) with $2 \%(\mathrm{v} / \mathrm{v})$ glutaraldehyde $(0.5 \mu 1)$ on the surface of glassy carbon disc (diameter: $3 \mathrm{~mm}$, TOKAI CARBON, GC-20). Soy sauce, Marinarge 18\% (M-18, MITSUBISHI Gas Chem. Co.) and its HPLC data on L-amino acid components were provided from TANNO Fisheries Co. . The amino acid analysis was performed by using PICO TAG amino acid analyzer (Waters). 2-Ferrocenylethanol (2-FEA) was synthesized according to the literature 2). The amperometric measurements were carried out at a controlled potential $(0.20 \mathrm{~V}$ vs. SCE). All the experiments were conducted in $0.05 \mathrm{M}$ phosphate buffer $(\mathrm{pH} 7.5)$ at $30^{\circ} \mathrm{C}$.

\section{RESULTS AND DISCUSSION}

We have used 2-FEA as the mediator, taking account of the electrochemical reversibility, stability and redox potential. Other redox species such as Vitamin $K_{3}$ could also be used as the mediator ${ }^{3)}$. Fig. 1 depicts the cyclic voltammograms for $0.2 \mathrm{mM}$ 2-FEA on the $\mathrm{Dp}+\mathrm{GluDH}$ immobilized electrode. 2-FEA exhibits reversible behavior with a half-wave potential of $0.15 \mathrm{~V}$ vs. SCE (solid line). The addition of $0.5 \mathrm{mM} \mathrm{NADH}$ to the solution results in an obvious increase in the peak current and the appearance of a prewave at ca. $0.05 \mathrm{~V}$. This phenomenon demonstrates 


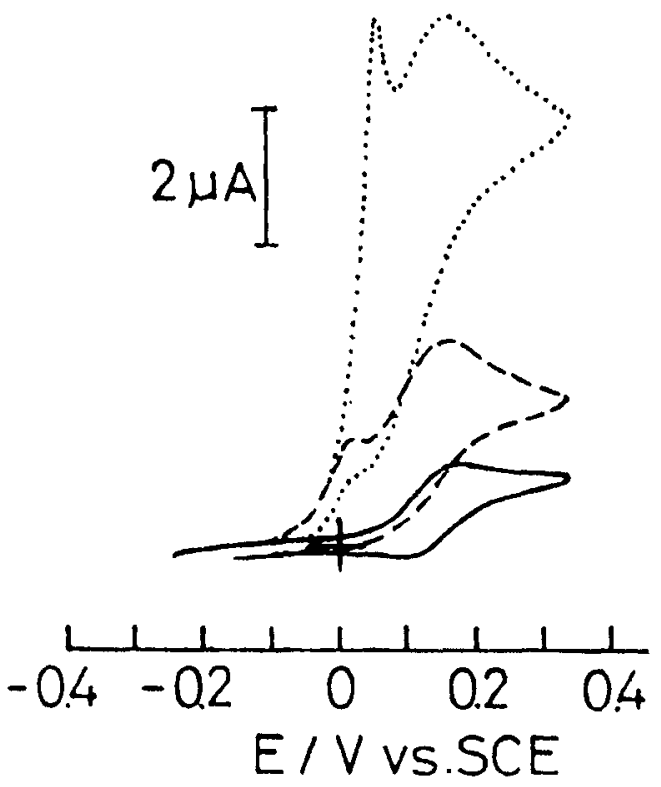

Fig. 1 Cyclic voltammograms for $0.2 \mathrm{mM} \mathrm{2-FEA} \mathrm{on}$ the $\mathrm{Dp}+\mathrm{GluDH}$ coimmobilized electrode.

- : Without addition. ---- : With $0.5 \mathrm{mM} \mathrm{NADH}$.

..... : With $0.5 \mathrm{mM} \mathrm{NADH}$ and $1 \mathrm{mM}$ L-glutamate in $0.05 \mathrm{M}$ phosphate buffer (pH 7.5).

Scan rate: $10 \mathrm{mV} \mathrm{s}^{-1}$. Temperature: $30^{\circ} \mathrm{C}$.

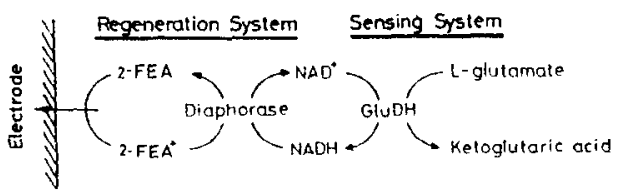

Fig. 2 Schematic representation of catalytic oxidation of L-glutamate at the $\mathrm{Dp}+\mathrm{GluDH}$ immobilized electrode.

that Dp effectively catalyzes the oxidation of NADH by the oxidized 2-FEA 4). When $1 \mathrm{mM} \mathrm{L}$ glutamate was added to the same solution, these peak currents showed further increase, indicating that the Dp and GluDH immobilized on the electrode surface act effectively as the cooperative catalysts for the oxidation of L-glutamate (Fig. 2). We have carried out the amperometric measurements at $0.20 \mathrm{~V}$ vs. SCE under stirring. The current response upon addition of $L$ glutamate was rapid and the steady state current was obtained within $30 \mathrm{~s}$. The linearity of the relationship was observed over the concentration range, 5-200 $\mu \mathrm{M}$. The $\mathrm{Dp}+\mathrm{AlaDH}$ and $\mathrm{Dp}$
+ LeuDH coimmobilized electrodes also showed responses upon addition of $\mathrm{L}$-alanine and $\mathrm{L}$ leucine, respectively, and the responses increased linearly with the concentration in the range of 5 $150 \mu \mathrm{M}$. We have applied the present electrodes to determination of individual L-amino acid in a M-18 soy solution. The concentrations of $\mathrm{L}$ glutamate, L-alanine and L-leucine determined by the present sensors show good agreements with those obtained by an amino acid analyzer based on HPLC (Table 1). Near $100 \%$ recovery data were observed in recovery tests.

In conclusion, the present method provides a simple way to detect individual L-amino acid at a micromolar concentration level. Optimization of the concentration of mediator and coenzyme, and further refinement of the electrodes connecting with a flow injection system will result in a convenient system for electrochemical detection of individual L-amino acid in routine operation.

Table 1 Concentration of L-amino acids in a soy sauce.

\begin{tabular}{|c|c|c|c|}
\hline & \multicolumn{3}{|c|}{ Concentration/mM } \\
\hline & $\begin{array}{c}\text { L- } \\
\text { glutamate }\end{array}$ & $\begin{array}{c}\text { L- } \\
\text { alanine }\end{array}$ & $\begin{array}{c}\text { L- } \\
\text { leucine }\end{array}$ \\
\hline Enzyme sensor ${ }^{\text {a) }}$ & 48.0 & 72.0 & $152.0^{\mathrm{b})}$ \\
HPLCc) $^{\text {c) }}$ & 50.7 & 73.2 & $\begin{array}{c}52.6 \\
\left(53.1^{\mathrm{d})}, 38.1^{\mathrm{e})}\right)\end{array}$ \\
\hline
\end{tabular}

a) reproducibility; ca. $5 \%$.

b) $\mathrm{Dp}+$ LeuDH electrode responds to L-leucine, Lvaline and $\mathrm{L}$-isoleucine.

c) Data from Tanno Fisheries Co. .

d) L-valine. e) L-isoleucine.

\section{REFERENCES}

1) M. Nanjo, G.G. Guilbault, Anal. Chim. Acta, 73, 367 (1974).

2) D. Lednicer, J.K. Lindsay, and C.R. Hanser. J. Org. Chem., 23,653 (1958).

3) K. Miki, T. Ikeda, S. Todoriki, and M. Senda, Anal. Sci., 5, 269 (1989).

4) T. Matsue, H. Yamada, H.-C. Chang, and I. Uchida, K. Nagata, and K. Tomita. Biochim. Biophys. Acta, 1038, 29 (1990). 